\title{
Role of Vertical Jumps and Anthropometric Variables in Maximal Kicking Ball Velocities in Elite Soccer Players
}

\author{
by \\ Lois Rodríguez-Lorenzo ${ }^{1}$, Miguel Fernandez-del-Olmo ${ }^{1}$, \\ José Andrés Sanchez-Molina ${ }^{1}$, Rafael Martín-Acero ${ }^{1}$
}

\begin{abstract}
Kicking is one of the most important skills in soccer and the ability to achieve maximal kicking velocity with both legs leads to an advantage for the soccer player. This study examined the relationship between kicking ball velocity with both legs using anthropometric measurements and vertical jumps (a squat jump (SJ); a countermovement jump without (CMJ) and with the arm swing (CMJA) and a reactive jump (RJ)). Anthropometric measurements did not correlate with kicking ball velocity. Vertical jumps correlated significantly with kicking ball velocity using the dominant leg only ( $r=.47, r=.58, r=.44, r=.51$, for SJ, CMJ, CMJA and RJ, respectively). Maximal kicking velocity with the dominant leg was significantly higher than with the non-dominant leg $(t=18.04, p<0.001)$. Our results suggest that vertical jumps may be an optimal test to assess neuromuscular skills involved in kicking at maximal speed. Lack of the relationship between vertical jumps and kicking velocity with the non-dominant leg may reflect a difficulty to exhibit the neuromuscular skills during dominant leg kicking.
\end{abstract}

Key words: kicking performance, jumping performance, strength, dominant leg, non-dominant leg, kicking deficit.

\section{Introduction}

Soccer is one of the most popular sports in the world with 265 million players worldwide (FIFA, 2007). Different studies have investigated match skills in soccer (Lees et al., 1998; Masuda et al., 2005).

Kicking is the most widely studied soccer skill (De Proft et al., 1988; Masuda et al., 2005; Rodríguez-Lorenzo et al., 2015), as it is one of the most important skills in soccer (Bacvarevic et al., 2012; Barfield et al., 2002). Moreover, kicking is the most used and determinant skill in competition (Kellis and Katis, 2007; Sedano et al., 2009).

The performance of soccer kicking usually depends on kicking ball velocity and accuracy (Lees and Noland, 1998). Although, accuracy is an important factor, kicking performance in soccer has been evaluated predominantly by maximum ball velocity (Markovic et al., 2006). Therefore, when a soccer player performs an accurate kick towards an area of difficult interception by the goalkeeper, the chance of scoring increases with an increased ball velocity since there is less time for the goalkeeper to react (Markovic et al., 2006). In addition, it has been shown that the most successful goal scorers are those players who are able to score with both legs (Starosta, 1988). Therefore, the ability to kick with both legs leads to an advantage for the soccer player. However, the majority of the players have a slower maximal ball velocity when they kick with the nondominant leg in comparison with the dominant leg (Barfield, 1995; Barfield et al., 2002; Dörge et al., 2002; Nunome et al., 2006a).

Several studies have been conducted in order to explore different factors that could affect maximal kicking velocity (Rodríguez-Lorenzo et al., 2015). Maximum kicking velocity is a complex

1 - Learning and Human Movement Control Group, University of A Coruña, A Coruña, Spain. 
skill that depends on technical (Juárez et al., 2010; Nunome et al., 2006b), biomechanical (Young and Rath, 2011), physiological (Ferraz et al., 2012), neuromuscular (Dörge et al., 1999; Kristensen et al., 1996; Nunome et al., 2006a) and strength factors (Masuda et al., 2005). In addition, anthropometric measurements have been studied in the context of potential use for the selection and future development of young soccer players (Helsen et al., 2005; Wong et al., 2009). Although, a positive relationship has been found between kicking performance and anthropometric measurements (Alessandro et al., 2014; Luhtanen, 1988), no studies have been conducted with adult elite soccer players. Therefore, it remains unknown whether the anthropometric variables are related with kicking performance once the players complete their physical growth. Improvement in maximal kicking velocity may be mediated by any one of the above mentioned factors. For instance, an increase in maximum kicking velocity performance may be attributed to an improvement in strength and explosiveness of the leg extensor muscles (Maulder and Cronin, 2005). However, the relationship between strength measurements and kicking performance is a subject of controversy (Young and Rath, 2011).

One way to determine the potential importance of the function of various muscles is to identify the relationship between strength measures and maximum ball velocity (Young and Rath, 2011). Some investigators reported positive and significant correlations between isokinetic strength and maximum kicking velocity (Luhtanen, 1988; Masuda et al., 2005). However, other studies did not find that relationship (Dörge et al., 2002; McLean and Tumilty, 1993; Mognoni et al., 1994; Narici et al., 1988). Therefore, there is a lack of consistency in the relationship between isokinetic strength and maximum kicking velocity, and thus, it is not possible to establish a cause-and-effect relationship between the strength measures and kicking performance (Young and Rath, 2011).

An alternative to isokinetic tests could be vertical jump tests. Explosive strength in the form of vertical jumping has been deemed to be functional for optimal performance in soccer (Stolen et al., 2005). However, only a few studies have previously investigated the possible relationship between vertical jump variables and maximal kicking velocity. Furthermore, the results from these studies are inconsistent. While some studies reported significant correlations between vertical jump variables and maximal kicking performance (Clark and Brooks, 2011), others found unclear (Juárez et al., 2008; Sousa et al., 2003) or non-significant correlations (Juárez et al., 2008, 2010). Moreover, these studies were conducted in students and children and thus, it remains unknown whether performance in the vertical jump is associated with maximal kicking velocity in elite soccer players and whether that relationship is similar for both the dominant and non-dominant leg.

Therefore, the goals of this study were as follows: i) to explore the relationship between vertical jumps and maximal kicking ball velocity with both legs in elite soccer players, ii) to compare the kicking performance between both legs, and iii) to explore the relationship between maximal kicking ball velocity and anthropometric measurements. This study provides further information regarding the role of strength in maximal kicking velocity, the nature of the kicking deficit of the non-dominant leg and the effect of the anthropometric features in kicking velocity.

\section{Material and Methods}

\section{Experimental Approach to the Problem}

Two experimental sessions were conducted on 2 days, separated by 48 hours of rest, at an ambient temperature of 16 to $18^{\circ} \mathrm{C}$. Four familiarization sessions were conducted where subjects practiced maximal kicking and jump tests, in order to minimize any learning or habituation effects. For vertical jumps we followed the procedure described by Acero et al. (2011). To avoid inter-observer variability, the same experienced investigator tested all subjects.

The first experimental session started with the anthropometric measurements, followed by a warm-up which consisted of $5 \mathrm{~min}$ indoor running at a self-selected pace, a $5 \mathrm{~min}$ active stretching protocol, mainly for the lower limbs, 2 submaximal squat jumps and 2 submaximal countermovement jumps. After the warm-up, subjects were demonstrated each of the tests and performed 1 practice trial for habituation. The following 2 trials were recorded as experimental trials. Each subject had at least $3 \mathrm{~min}$ of rest 
between 2 consecutive trials on the vertical jumps in order to avoid fatigue.

The second experimental session consisted of the maximal kick test. The session started with a warm-up consisting of 5 min indoor running at a self-selected pace, a $5 \mathrm{~min}$ active stretching protocol, mainly for the lower limbs, 6 submaximal and 2 maximal instep kicks performed with each leg. After warming-up the kick test was demonstrated, followed by 1 practice trial performed with each leg. The following 3 trials with both the preferred and the non-preferred leg were recorded as experimental trials. The trial that produced the highest speed for both the preferred and the non-preferred leg was selected for further analysis. The order of testing was randomized between the preferred and the non-preferred leg. Each subject had at least $1 \mathrm{~min}$ of rest between 2 consecutive trials to avoid fatigue.

\section{Participants}

Twenty three male elite soccer players (mean age $20.27 \pm 4.49$ years; body mass $71.84 \pm$ $6.52 \mathrm{~kg}$; body height $177.3 \pm 5.83 \mathrm{~cm}$; body mass index $22.84 \pm 1.2 \mathrm{~kg} \cdot \mathrm{m}^{-2}$; goalkeepers $\mathrm{n}=2$, defenders $n=7$, midfielders $n=7$, and attackers $n$ = 7) from the Real Club Deportivo de la Coruña second team (3rd Spanish Division), were tested as a part of their athletic training program during the final month of the 2013-2014 competitive season (May, 2014).

At the time of the study, all players had an average of over 10 years of soccer training experience and had trained an average of 4 times, as well as one game per week for at least 4 years. Fifteen players preferred to kick with the right leg and eight preferred to kick with the left leg.

After receiving a detailed explanation of the study's benefits and risks, all participants signed informed consent and completed a form giving, personal, medical and training details. None of the subjects reported neurological diseases or recent injuries. The study was approved by the medical department of Real Club Deportivo de la Coruña (Spain). The experimental procedures of the study were carried out in accordance with the Declaration of Helsinki.

\section{Procedures}

Anthropometric measurements

A total of eleven anthropometric measurements were recorded according to the
International Society for the Advancement of Kinanthropometry's protocol (Marfell-Jones et al., 2006). All anthropometric measurements were taken twice in the right side of the body by the same trained anthropometrist in standardized order after a proper calibration of the measuring instruments.

Body mass was measured using a BFW300 Platform scale (Adam Equipment Co. Ltd., UK.) to the nearest $0.1 \mathrm{~kg}$. Body stature was measured using a Harpenden stadiometer (Holtain Limited, UK.) to the nearest $0.1 \mathrm{~cm}$. Lengths and breadths were measured using a Siber-Hegner Anthropometer (Zurich, Switzerland) to the nearest $0.1 \mathrm{~cm}$. Girths were measured using a Lufkin W606PM flexible steel tape (Cooper Industries, USA) to the nearest $0.1 \mathrm{~cm}$.

Kicking test

A modified version of the kicking test described by Markovic et al. (2006) was used to measure maximal ball velocity. The participants were instructed to perform an instep kick of a stationary ball of standard size and standard inflation (Fédération Internationale de Football Association, FIFA, standard) as fast as possible towards the radar gun. To standardize the procedure, the participants were restricted to a 5step run-up from a position directly behind the ball. They were specifically instructed to focus only on maximum kicking velocity and the trials that missed the entire target area were repeated. Only 6 players needed to perform additional kicks and the maximum number of kicks performed by a player was 8 .

Kicking performance was determined from maximal ball velocity. Velocity, expressed in $\mathrm{m} / \mathrm{s}$, was assessed using a stationary Doppler radar gun (Stalker Sport 2, Stalker Radar, Plano, Texas, USA) that can measure speeds between $2.23 \mathrm{~m} \cdot \mathrm{s}^{-1}$ and $67.04 \mathrm{~m} \cdot \mathrm{s}^{-1}$ with accuracy of \pm 0.045 $\mathrm{m} \cdot \mathrm{s}^{-1}$. The radar gun (operating frequency of $24.125 \mathrm{GHz}$ ) was attached to a $0.7 \mathrm{~m}$ high stand and positioned behind a net, approximately $5 \mathrm{~m}$ from the starting position of the ball. The size of the net was sufficient to cover all kicks that deviated less than $15^{\circ}$ from the direction of the radar gun (Markovic et al., 2006). The radar gun was always calibrated immediately before the sessions according to the instructions given in the user's manual. 
Vertical Jump Tests

The SJ and CMJ tests were performed according to the protocols described by Bosco et al. (1983). The CMJA and 1RJA tests were performed according to the protocols described by Acero et al. (2012), where a more restricted action of the arms was imposed compared with the protocols of Bosco et al. (1983).

All vertical jumps were performed on a force plate (Quattro Jump, Kisler, Winterhur, UK) in the following order: a squat jump (SJ), a countermovement jump (CMJ), a countermovement jump with the arm swing (CMJA) and a reactive jump with the arm swing (1RJA).

\section{Data Analyses}

Anthropometric variables consisted of eleven measurements: body mass, body stature, 2 lengths (trochanterion and tibiale laterale height), 3 girths (arm flexed and tensed girth; thigh girth; calf girth), and 4 breadths (biiliocristal; biepicondylar femur). The mean value of the 2 measurements at each site was used for the analyses.

For the kicking test average velocity, maximal velocity and the coefficient of variation $(\mathrm{CV})$ from the 3 trials were calculated. In addition, a kicking deficit variable (KD) was calculated according to the following formula:

$$
K D=\left(\frac{\text { KVdomMax }- \text { KVnodomMax }}{K V \text { domMax }}\right) \cdot 100
$$

where KVdomMax and KVnodommax are the maximal velocities achieved with the dominant and non-dominant leg, respectively. The kicking deficit was defined as the percentage of the difference between maximal velocity obtained with the non-dominant leg in relation with the dominant leg.

For the vertical jump, height was calculated according to the following formula (Bosco et al., 1983):

$$
\text { SJ_H }=0.5 \cdot g \cdot\left(\frac{\text { flight time }}{2}\right)^{2}
$$

where flight time was the time between the takeoff and landing.

\section{Statistical Analyses}

Normal distribution of the variables was tested using the Shapiro-Wilk W-test. A Student's $t$-test for dependent samples was performed to compare maximal kicking velocities and $\mathrm{CVs}$ obtained with the dominant and non-dominant leg.

In order to explore whether the kicking deficit was related to maximal kicking velocity achieved by the dominant or non-dominant leg (i.e. to test whether the players with the highest maximal kick velocity with the dominant leg also had the greatest kicking deficit), the sample was divided into two groups according to the median value of the kicking deficit velocity. A Student's $t$ test for independent samples was performed for KVdomMax and KVnodomMax.

Pearson product correlation analysis was performed to assess the relationships between the kick test (KVdomMax, KVnodomMax), anthropometric measurements and the vertical jumps (SJ, CMJ, CMJA, RJ).

All statistical analyses were performed using SPSS version 21 (SPSS, Chicago, IL). A $p$ value $<0.05$ was considered statistically significant.

\section{Results}

Mean and SD values of the variables are shown in Table 1. None of the data violated the normality assumption necessary to conduct parametric statistical tests.

T-test analysis revealed that maximal kicking velocity with the dominant leg (KVdomMax) was significantly higher than with the non-dominant leg $(t=18.04, p<0.001)$. No significant differences were found for the individual CV values of the kicking velocity between the legs.

There were no differences in the KVdomMax and KVnodomMax between the groups with higher and lower kicking deficits, suggesting no relationship between maximal kicking velocity achieved by either leg and kicking velocity.

Table 2 shows the coefficients of correlation among variables. Anthropometric measurements did not correlate with kicking velocities. Maximum kicking velocities between legs correlated significantly $(r=.75, p<0.01)$. 
Table 1

Characteristics of the sample

\begin{tabular}{lcccc}
\hline \multicolumn{1}{c}{ Dimension } & Mean & SD & Minimum & Maximum \\
\hline Age (years) & 21.12 & 2.42 & 18.03 & 26.31 \\
Body mass (kg) & 71.84 & 6.52 & 61.20 & 86.20 \\
Stature (cm) & 177.23 & 5.83 & 167.50 & 189.00 \\
Trochanterion height (cm) & 90.91 & 4.00 & 83.30 & 99.70 \\
Tibiale laterale height (cm) & 43.92 & 2.63 & 39.50 & 49.20 \\
Arm flexed and tensed girth (cm) & 31.62 & 2.04 & 27.70 & 35.50 \\
Thigh girth (cm) & 51.04 & 3.84 & 42.50 & 57.60 \\
Calf girth (cm) & 36.00 & 2.07 & 30.50 & 39.00 \\
Biiliocristal breadth (cm) & 28.97 & 1.27 & 26.80 & 31.20 \\
Maximum velocity Dominant leg (m/s) & 31.48 & 1.45 & 29.17 & 34.5 \\
Maximum velocity Non-Dominant leg (m/s) & 27.54 & 1.53 & 24.72 & 30.72 \\
CV Average velocity Dominant leg (m/s) & 1.03 & 0.74 & 0.30 & 3.49 \\
CV Average velocity Non-Dominant leg (m/s) & 1.36 & 1.19 & 0.03 & 4.82 \\
Kicking Deficit & 12.49 & 3.24 & 5.52 & 17.59 \\
SJ (cm) & 31.10 & 4.34 & 21.60 & 38.20 \\
CMJ (cm) & 33.07 & 3.88 & 23.90 & 38.70 \\
CMJA (cm) & 37.79 & 3.94 & 29.00 & 45.00 \\
RJ (cm) & 31.68 & 7.37 & 16.40 & 44.40 \\
\hline
\end{tabular}

\section{Table 2}

Correlation values between variables.

\begin{tabular}{lcc}
\hline \multicolumn{1}{c}{ Variables } & $\begin{array}{c}\text { Maximum velocity } \\
\text { Dominant leg }\end{array}$ & $\begin{array}{c}\text { Maximum velocity } \\
\text { Non-Dominant leg }\end{array}$ \\
\hline Body mass & Anthropometric measurements & \\
Stature & 0.274 & 0.157 \\
Trochanterion height & 0.287 & 0.174 \\
Tibiale laterale height & 0.029 & 0.021 \\
Arm flexed and tensed girth & -0.099 & -0.136 \\
Thigh girth & 0.329 & 0.323 \\
Calf girth & 0.035 & 0.103 \\
Biiliocristal breadth & 0.252 & 0.293 \\
& 0.226 & 0.281 \\
Maximun velocity Dominant leg & Kicking test & $0.754^{* *}$ \\
Maximun velocity Non-Dominant leg & - & - \\
& $0.754^{* *}$ & 0.075 \\
SJ & Jump test & 0.312 \\
CMJ & $.475^{*}$ & 0.317 \\
CMJA & $.582^{* *}$ & 0.112 \\
RJ & $.444^{*}$ & $.510^{*}$ \\
\hline
\end{tabular}

* Correlations significant at $p<0.05 ;{ }^{* *}$ Correlations significant at $p<0.01$. 
Table 3

Kicking deficit and maximal kicking velocity values for dominant and non-dominant leg performance

\begin{tabular}{|c|c|c|c|c|c|c|c|c|}
\hline \multirow[b]{2}{*}{ Research } & \multicolumn{3}{|c|}{ Subject characteristics } & \multirow[b]{2}{*}{ Kick } & \multirow{2}{*}{$\begin{array}{l}\text { Kicking } \\
\text { deficit }+\end{array}$} & \multirow[b]{2}{*}{ RI } & \multirow{2}{*}{$\begin{array}{l}\mathrm{VB}\left(\mathrm{m} \cdot \mathrm{s}^{-1}\right) \\
\text { Dominant }\end{array}$} & \multirow{2}{*}{$\begin{array}{l}\mathrm{VB}\left(\mathrm{m} \cdot \mathrm{s}^{-1}\right) \\
\text { Non- } \\
\text { dominant }\end{array}$} \\
\hline & $\mathrm{n}$ & $\begin{array}{l}\text { Age } \\
\text { Instep }\end{array}$ & Player level & & & & & \\
\hline $\begin{array}{l}\text { Narici et al. } \\
(1988)\end{array}$ & 11 & $25.1 \pm 5.0$ & Amateur & & 11.5 & ARS & $20.0 \pm 3.6^{* * *}$ & $17.7 \pm 2.2^{* * *}$ \\
\hline $\begin{array}{l}\text { McLean and } \\
\text { Tumilty (1993) }\end{array}$ & 20 & $16.8 \pm 0.7$ & Elite junior & Drive kick & 16.45 & $\mathrm{R}$ & $21.95 \pm 1.67^{*}$ & $18.34 \pm 1.39^{*}$ \\
\hline $\begin{array}{l}\text { Mognoni et al. } \\
\text { (1994) }\end{array}$ & 24 & NA & Junior & & 9.32 & & $23.6 \pm 2.5$ & $21.4 \pm 2.6$ \\
\hline $\begin{array}{l}\text { Barfield. } \\
\text { (1995) }\end{array}$ & 18 & NA & Expert & & 7.95 & $\mathrm{R}$ & $26.4 \pm 2.1$ & $24.3 \pm 2.0$ \\
\hline $\begin{array}{l}\text { Dörge et al. } \\
\text { (2002) }\end{array}$ & 30 & & Skilled & & 12.95 & $\mathrm{R}$ & $24.7 \pm 2.5^{*}$ & $21.5 \pm 2.0^{*}$ \\
\hline \multirow{3}{*}{$\begin{array}{l}\text { Barfield et al. } \\
\text { (2002) }\end{array}$} & 8 & $19-22$ & Élite & Instep & & $2 \mathrm{C}$ & $* * *$ & $* * *$ \\
\hline & 2 & & Male & & 6.71 & & $25.3 \pm 1.51^{* * *}$ & $23.6 \pm 1.57^{* * *}$ \\
\hline & 6 & & Female & & 12.09 & & $21.5 \pm 2.44^{* * *}$ & $18.9 \pm 2.05^{* * *}$ \\
\hline $\begin{array}{l}\text { Vaverka et al. } \\
\text { (2003) }\end{array}$ & 12 & $15.7 \pm 0.4$ & Skilled & & 15.14 & $3 \mathrm{D}$ & $27.68 \pm 1.32$ & $23.49 \pm 2.05$ \\
\hline $\begin{array}{l}\text { Nunome } \\
\text { (2006a) }\end{array}$ & 5 & $16.8 \pm 0.4$ & Skilled & Instep & 15.58 & $3 C$ & $32.1 \pm 1.7^{*}$ & $27.1 \pm 1.2^{*}$ \\
\hline \multirow{2}{*}{$\begin{array}{l}\text { Sedano et al. } \\
(2009 b)\end{array}$} & \multirow{2}{*}{10} & \multirow{2}{*}{$22.8 \pm 2.1$} & \multirow{2}{*}{ Elite female } & Pre-Trained & 16.45 & \multirow{2}{*}{$\mathrm{R}$} & $19.45 \pm 0.7^{*}$ & $16.25 \pm 0.45^{*}$ \\
\hline & & & & Post-Trained & 15.45 & & $21.75 \pm 0.58^{*}$ & $18.39 \pm 0.64^{*}$ \\
\hline \multirow{5}{*}{$\begin{array}{l}\text { Berjan et al. } \\
(2012)\end{array}$} & 106 & 11.514 .5 & Elite young & Instep & & $\mathrm{R}$ & & \\
\hline & 27 & $12.2 \pm 0.3$ & & Maximal & 13.21 & & $22.7 \pm 2.5^{*}$ & $19.7 \pm 2.3^{*}$ \\
\hline & 26 & $13.1 \pm 0.2$ & & Maximal & 18.15 & & $24.8 \pm 2.1^{*}$ & $20.3 \pm 2.7^{*}$ \\
\hline & 26 & $14.3 \pm 0.3$ & & Maximal & 14.76 & & $27.1 \pm 2.8^{*}$ & $23.1 \pm 2.9^{*}$ \\
\hline & 27 & $15.3 \pm 0.3$ & & Maximal & 14.63 & & $28.7 \pm 2.3^{*}$ & $24.5 \pm 3.1^{*}$ \\
\hline $\begin{array}{l}\text { Barbieri et al. } \\
\text { (2015) }\end{array}$ & 10 & $21.8 \pm 2.2$ & $\begin{array}{l}\text { Amateur } \\
\text { futsal }\end{array}$ & Instep & 10.91 & $3 \mathrm{D}$ & $24.27 \pm 2.21^{* * *}$ & $21.62 \pm 2.26^{* * *}$ \\
\hline
\end{tabular}

Note: $n=$ Sample size; $R I=$ Register instrument; $R=$ Radar;

$A R S=A$ udio recording system $; C=$ Video camera $3 D=$ Motion capture system;

$V B=$ Kicking ball velocity; $N A=$ No available data;

Statistical significance: ${ }^{*} p<0.05,{ }^{* *} p<0.01,{ }^{* * *} p<0.00$;

+ Kicking deficit is calculated from the total average reported by those studies

The results obtained from vertical jump tests revealed a significant and moderate relationship between maximum kicking velocity with the dominant leg and the height of each vertical jump (SJ, CMJ, CMJA, RJ). Interestingly, maximum kicking velocity with the non-dominant leg did not correlate with any vertical jump.

\section{Discussion}

The purpose of this study was to compare maximum kicking velocity between the dominant and non-dominant leg and their relationship with the performance of a vertical jump. The main finding of our study was that there was a significant correlation between the performance of a jump test and maximum kicking velocity with the dominant leg, while no significant relationship was found for the non-dominant leg. In addition, maximum ball velocity after a soccer kick was significantly different between the dominant and 
non-dominant leg, indicating a kicking deficit with the non-dominant leg.

Ball velocity of maximal instep kicks for the dominant and non-dominant leg had been previously studied (Table 3 ). Ball velocity values of the dominant (mean $31.48 \pm 1.45 \mathrm{~m} / \mathrm{s}$ ) and nondominant (mean $27.54 \pm 1.53 \mathrm{~m} / \mathrm{s}$ ) leg recorded in the present study were higher than in previous studies (mean 14.7). This may be due to the fact that our subjects were elite soccer players with at least 10 years of extensive practice.

We found a significant relationship between the height achieved in the vertical jump tests and maximum kicking velocity with the dominant leg. To the best of our knowledge only two studies had shown a significant relationship between maximal kicking velocity with the dominant leg and vertical jumps (Sousa et al., 2003). Sousa et al. (2003) reported a very weak significant relationship between kicking velocity and the SJ $(\mathrm{r}=.10)$ and the CMJ $(\mathrm{r}=.07)$. It is possible that the wider range of values during the vertical jumps in the Sousa's study in comparison with our study could explain the low relationship that the authors found between both variables. Nevertheless, our results contrast with previous studies that have shown no relationship between ball velocity and performance in different jump tests (Juárez et al., 2008, 2010). Several methodological issues, such as familiarization with the testing protocols or the features of the sample, may account for these differences. In our study the subjects performed four preliminary familiarization sessions of the testing procedures in order to minimize any effects of learning, achieve their maximal performance during the experimental sessions, and obtain reliable data. No familiarization sessions were conducted in previous studies (Juárez et al., 2008, 2010; Sousa et al., 2003). It is possible that the lack of familiarization sessions could increase the intra-subject variability (Acero et al., 2011), affecting the correlation analysis. In addition, the sample used in previous studies was very small, the soccer players were non-elite and under 15 years old (Juárez et al., 2008). The elite level and years of practice in the current study's sample mean that the data are reliable, as recent studies have reported that experienced players displayed less variability in the kicking pattern (Southard, 2014). In summary, it is likely that the rigorous methodology used in our study for data acquisition together with the expertise of the soccer players contributed to the strong correlations observed between the vertical jump and kicking performances.

It is also plausible that, in the current study, the core strength training program that the soccer players performed as a part of their regular soccer training may have contributed to the performance of both the ball kick and the vertical jump and thus, enhanced the correlation between these variables. This is supported by a recent study which showed that core training improved maximum kicking velocity due to a better transfer of forces from the trunk to limb muscles (Prieske et al., 2015). In addition, performance of a vertical jump is also enhanced by trunk stability exercises (Butcher et al., 2007).

The observed correlation between the performance of the vertical jumps and ball kicking with the dominant leg suggests that both tasks share some common muscle demands, such as the ability to reach high levels of force in a short period of time. The vertical jump test could be an optimal test to assess the neuromuscular skills involved when kicking at maximal speed. This hypothesis is supported by previous studies that have reported similar improvements in kicking velocity and the height of vertical jumps after strength training programs (García-Pinillos et al., 2014; Marques et al., 2013; Sedano et al., 2009). Interestingly, no correlations were found between the vertical jumps and kicking velocity with the non-dominant leg. This lack of the relationship could be interpreted as a difficulty to utilize the neuromuscular skills required for an optimal kicking performance. Higher ball velocity can be achieved by increasing foot velocity at impact (Young and Rath, 2011), which is dependent on the neuromuscular skill and technique levels (Dörge et al., 1999; Katis and Kellis, 2010; Kellis et al., 2004; Southard, 2014). It is possible that in our players the muscular function of each leg was highly specific. While the non-dominant leg is trained to develop isometric tension (to provide a stable platform for the swing of the kicking leg), the kicking and dominant leg is characterized by an explosive action (Dörge et al., 2002; Lees and Noland, 1998; Young and Rath, 2011). This muscle specificity could be compromised during the kicking action with the non-dominant leg, which 
may explain the lack of correlation with the vertical jump, a skill that requires an explosive muscle contraction.

Our results show that subjects produced significantly higher ball velocity with their preferred leg than with their non-preferred leg. Similar findings have been shown in expert soccer players (Barfield, 1995, 2002), amateurs (Marques et al., 2011; Mognoni et al., 1994; Narici et al., 1988; Vaverka, 2003), young subjects (Berjan et al., 2012), for both genders (Barfield et al., 2002; Sedano et al., 2009), for kicks with or without accuracy demand (Bacvarevic et al., 2012), and for different techniques and kicking conditions (Marques et al., 2011; McLean and Tumilty, 1993). The most straightforward explanation for these results is that the kicking technique is better with the dominant compared to non-dominant leg (Barbieri et al., 2015; Barfield et al., 2002; Teixeira, 1999; Vaverka et al., 2003). Unfortunately, in the current study we did not record the biomechanics of the kick in order to test this hypothesis. However, the coefficient of variation of the kicking performance was not significantly different between legs. It is known that the expertise of a task is related to lower variability in performance (Schmidt, 1988). Thus, it is possible that the specificity of the muscle function of each leg during kicking could be a possible reason for the difference in kicking velocity observed between dominant and non-dominant legs.

In our study the players who achieved higher ball velocities with the dominant leg, also achieved higher ball velocities with the nondominant leg. However, when the players were divided into two groups according to the median value of the kicking deficit, there were no differences between kicking velocities with the dominant and non-dominant leg. These results suggest that the kicking deficit is independent of the absolute maximal kicking velocity of the dominant leg. In addition, the values of the kicking deficit were more variable across players compared with the values for maximal kicking velocity for both dominant and non-dominant legs. For instance, the player with the highest kicking deficit reported values threefold that of the player with the lowest deficit $(17.59 \%$ vs. $5.52 \%$, respectively). Therefore, the kicking deficit may be more helpful when evaluating and monitoring the individual capacity of each player to perform at similar levels with both legs rather than the use of absolute maximal velocity values. This is of relevance since the ability to kick with both legs is an advantage for the soccer player (Starosta, 1988).

The correlational analysis did not show any relationship between the anthropometric measurements and kicking velocity. This is in contrast with previous findings in young soccer players that had shown a significant correlation between kicking performance and body mass (Alessandro et al., 2014; Luhtanen, 1988) or between kicking performance and stature (Luhtanen, 1988). Lack of this relationship in the current study with adult elite soccer players suggests that anthropometric characteristics may be overvalued during the process of sports training, especially during puberty. Our results are in line with Alessandro et al. (2014) who found that anthropometric characteristics did not predict match-related technical performance and concluded that anthropometric features should be used with caution for talent identification or training purposes. However, it has been reported that soccer coaches select young players based on their anthropometric characteristics rather than their technical and tactical performance (Helsen et al., 2005; Maulder and Cronin, 2005). Such procedures could provide short-term benefits in soccer team performance, but may prevent the long-term development of young soccer players, since once players complete their physical growth value of the anthropometric advantages decreases and technical and tactical factors become significantly more important. Therefore, as suggested by Reilly et al. (2000) and Wong et al. (2009), psychological and soccer-specific skills should also be considered in the selection of young soccer players.

Our study has several limitations. The players were from a single soccer team and participated in the $3^{\text {rd }}$ Spanish Division, thus, extrapolation of our findings to other teams and soccer player's levels must be done with caution. In addition, the tests were conducted at the end of the competitive season and thus, it remains to be explored whether the relationships between variables found in the current study are consistent throughout the competitive season. Therefore, futures studies must address those questions in order to further understand the relationship 
between vertical jumps, anthropometric variables and maximal kicking ball performance.

Nevertheless, and from a practical point of view, the current study suggests that the kicking deficit variable may be useful for coaches when evaluating and monitoring the individual player's capacity to perform at similar levels with both legs rather than the use of the absolute maximal velocity. Finally, lack of a relationship between anthropometric variables and kicking performance supports the hypothesis that the anthropometric characteristics should not be overvalued by coaches during training and the selection process of soccer players.

\section{Conclusions}

In summary, our results show a positive relationship between the performance of vertical jumps and the dominant leg maximal kicking velocity. This suggests that vertical jumps may be an optimal test to assess the neuromuscular skills involved in kicking at maximal speed. This relationship was absent for the non-dominant leg and may be interpreted as a difficulty to utilize the neuromuscular skills required for an optimal kicking performance. Furthermore, our findings show that the kicking deficit exhibited by the nondominant leg is independent of the absolute maximal kicking velocities of the dominant leg.

\section{References}

Acero RM, Fernández-del Olmo M, Sánchez JA, Otero XL, Aguado X, Rodríguez FA. Reliability of squat and countermovement jump tests in children 6 to 8 years of age. Pediatr Exerc Sci, 2011; 23(1): 151-60

Acero RM, Sánchez JA, Fernández-del-Olmo M. Tests of Vertical Jump: Countermovement Jump With Arm Swing and Reaction Jump With Arm Swing. Strength Cond J, 2012; 34(6): 87-93

Alessandro H, Cattuzzo MT, Santos F, Monteiro C. Anthropometric characteristics, field test scores and match-related technical performance in youth indoor soccer players with different playing status. Int $J$ Perform Anal Spor, 2014; 14(2): 482-492

Bacvarevic B, Pazin N, Bozic R, Mirkov D, Kukolj M, Jaric S. Evaluation of a composite test of kicking performance. J Strength Cond Res, 2012; 26(7): 1945-1952

Barbieri FA, Gobbi LT, Santiago PR, Cunha SA. Dominant-non-dominant asymmetry of kicking a stationary and rolling ball in a futsal context. Journal of sports sciences, 2015; 33(13): 1411-1419

Barfield WR. Effects of selected kinematic and kinetic variables on instep kicking with dominant and nondominant limbs. J Hum Mov Stud, 1995; 29(6): 251

Barfield WR, Kirkendall DT, Yu B. Kinematic instep kicking differences between elite female and male soccer players. J Sports Sci Med, 2002; 1(3): 72

Bosco C, Luhtanen P, Komi PV. A simple method for measurement of mechanical power in Jumping. Eur J Appl Physiol, 1983; 50(2): 273-282

Butcher SJ, Craven BR, Chilibeck PD, Spink KS, Grona SL, Sprigings EJ. The effect of trunk stability training on vertical takeoff velocity. J Orthop Sports Phys Ther, 2007; 37(5): 223-231

Clark S, Brooks K. Relationship Between Soccer Specific Skills and Anthropometric Data in NCAA Division I Female Soccer Athletes. J Strength Cond Res, 2011; 25: S63-S64

Count FB. 270 million people active in football. FIFA Communications Division, Information Services 2007; 31

Dörge HC, Andersen TB, Sørensen H, Simonsen EB. Biomechanical differences in soccer kicking with the preferred and the non-preferred leg. J Sport Sci, 2002; 20(4): 293-299

Dörge HC, Andersen T, Sørensen H, Simonsen EB, Aagaard H, Dyhre-Poulsen P, Klausen K. EMG activity of the iliopsoas muscle and leg kinetics during the soccer place kick. Scand J Med Sci Sports, 1999; 9(4): 195-200

Ferraz R, Van Den Tillaar R, Marques MC. The effect of fatigue on kicking velocity in soccer players. J Hum Kinet, 2012; 35(1): 97-107

García-Pinillos F, Martínez-Amat A, Hita-Contreras F, Martínez-López EJ, Latorre-Román PA. Effects of a 
Contrast Training Program Without External Load on Vertical Jump, Kicking Speed, Sprint, and Agility of Young Soccer Players. J Strength Cond Res, 2014; 28(9): 2452-2460

Helsen WF, VanWinckel J, Williams AM. The relative age effect in youth soccer across Europe. J Sports Sci, 2005; 23(6): 629-636

Juárez D, López C, Navarro E. Analysis of the soccer kick and its relationship with the vertical Analysis of the soccer kick and its relationship with the vertical jump in young top-class soccer players. Rev Int Cienc Deporte, 2010; 6(19): 29-41

Juárez D, Valdivielso FN, Rubio RM, Ravé JM, Blázquez AA, Fernández-Arroyo VM. Relationship among maximal strength in squat exercise, jump, sprint and kicking ball performance. Rev Int Cienc Deporte, 2008; 4(10): $1-12$

Katis A, Kellis E. Three-dimensional kinematics and ground reaction forces during the instep and outstep soccer kicks in pubertal players. J Sports Sci, 2010; 28(11): 1233-1241

Kellis E, Katis A. Biomechanical characteristics and determinants of instep soccer kick. J Sports Sci Med, 2007; 6(2): 154

Kellis E, Katis A, Gissis I. Knee biomechanics of the support leg in soccer kicks from three angles of approach. Med Sci Sports Exerc, 2004; 36: 1017-28

Kristensen LB, Andersen TB, Sørensen H. Comparison of precision in the toe and instep kick in soccer at high kicking velocities. In T Reilly, J Cabri, D Araújo (eds): Science and Football V. London and New York: Routledge, 71; 2005

Lees A, Noland L. The biomechanics of soccer: a review. J Sports Sci, 1998; 16: 211-234

Luhtanen P. Kinematics and kinetics of maximal instep kicking in junior soccer players. "In Science and Football I (Edit by T. Reilly and other)." London: E. \& FN Spon, 441-448; 1988

Marfell-Jones M, Olds T, Stewart A, Carter L. International Standards for Anthropometric assessment. International Society for the Advancement of Kinanthropometry (ISAK). Potchefstroom: South Africa; 2006

Markovic G, Dizdar D, Jaric S. Evaluation of tests of maximum kicking performance. J Sports Med Phys Fitness, 2006; 46(2): 215-220

Marques MC, Pereira A, Reis IG, Tillaar RV. Does an in-Season 6-Week Combined Sprint and Jump Training Program Improve Strength-Speed Abilities and Kicking Performance in Young Soccer Players? J Hum Kinet, 2013; 39(1): 157-166

Marques MC, Pereira F, Marinho DA, Reis M, Cretu R. A comparison of ball velocity in different kicking positions with dominant and non-dominant leg in junior soccer players. Journal of Physical Education and Sport, 2011; 11(2): 49-56

Masuda K, Kikuhara N, Demura S, Katsuta S, Yamanaka K. Relationship between muscle strength in various isokinetic movements and kick performance among soccer players. J Sports Med Phys Fitness, 2005; 45(1): 44-52

Maulder P, Cronin J. Horizontal and vertical jump assessment: reliability, symmetry, discriminative and predictive ability. Phys Ther Sport, 2005; 6(2): 74-82

McLean BD, Tumilty DM. Left-right asymmetry in two types of soccer kick. Br J Sports Med, 1993; 27(4): 260262

Mognoni P, Narici MV, Sirtori MD, Lorenzelli F. Isokinetic torques and kicking maximal ball velocity in young soccer players. J Sports Med Phys Fitness, 1994; 34(4): 357-361

Narici MV, Sirtori MD, Mognoni P. Maximal ball velocity and peak torques of hip flexor and knee extensor muscles. "In Science and Football I (Edit by T. Reilly and other)." London: E. \& FN Spon, 429-433; 1988

Nunome H, Ikegami Y, Kozakai R, Apriantono T, Sano S. Segmental dynamics of soccer instep kicking with the preferred and non-preferred leg. J Sport Sci, 2006; 24(05): 529-541

Nunome H, Lake M, Georgakis A, Stergioulas LK. Impact phase kinematics of instep kicking in soccer. J 
Sport Sci, 2006; 24(1): 11-22

Prieske O, Muehlbauer T, Borde R, Gube M, Bruhn S, Behm DG, Granacher U. Neuromuscular and athletic performance following core strength training in elite youth soccer: Role of instability. Scand J Med Sci Sports, 2015; 26(1): 48-56

Reilly T, Williams AM, Nevill A, Franks A. A multidisciplinary approach to talent identification in soccer. J Sport Sci, 2000; 18(9): 695-702

Rodríguez-Lorenzo L, Fernandez-del-Olmo M, Martín-Acero R. A Critical Review of the Technique Parameters and Sample Features of Maximal Kicking Velocity in Soccer. Strength Cond J, 2015; 37(5): 26-39

Schmidt RA, Lee T. Motor Control and Learning. Human kinetics: Champaign Illions, 2; 1988

Sedano S, Vaeyens R, Philippaerts RM, Redondo JC, de Benito AM, Cuadrado G. Effects of lower-limb plyometric training on body composition, explosive strength, and kicking speed in female soccer players. J Strength Cond Res, 2009; 23(6): 1714-1722

Sousa P, Garganta J, Garganta R. Positional status, explosive strength and ball speed in kicking performance of young soccer players. Rev Port Cien Desp, 2003; 3(3): 27-35

Southard DL. Changes in kicking pattern: effect of experience, speed, accuracy, and effective striking mass. Res Q Exerc Sport, 2014; 85(1): 107-116

Sporiš G, Vučetić V, Jerković M. Relationship between kicking and sprinting performance. Int J Perform Anal Spor, 2007; 7(2): 28-35

Starosta W. Symmetry and asymmetry in shooting demonstrated by elite soccer players. Science and Soccer 1988; 346-355

Stolen T, Chamari K, Castagna C, Wisløff U. Physiology of soccer. Sports Med, 2005; 35(6): 501-536

Teixeira LA. Kinematics of kicking as a function of different sources of constraint on accuracy. Percept Mot Skills, 1999; 88(3): 785-789

Vaverka F, Janura M, Elfmark M. The velocity of soccer kicking and the laterality of the lower extremities. Analysis, 2003; 387: 103

Wong PL, Chamari K, Dellal A, Wisloff U. Relationship between anthropometric and physiological characteristics in youth soccer players. J Strength Cond Res, 2009; 23: 1204-1210

Young WB, Rath DA. Enhancing foot velocity in football kicking: the role of strength training. J Strength Cond Res, 2011; 25(2): 561-566

\section{Corresponding author:}

\section{Rafael Martín-Acero}

Departamento de Educación Física e Deportiva

Facultade de Ciencias do Deporte e a Educación Física, Universidade da Coruña.

Faculty of Sciences of Sport and Physical Education, University of A Coruña.

Av. Ernesto Che Guevara 121, Pazos-Liáns, 15179 Oleiros, A Coruña, Spain.

Phone: +34 981167000,

Fax: +34 981167048,

E-mail: address: maracero@udc.es 